\title{
Belgeo
}

Revue belge de géographie

2| 2021

Dynamiques des campagnes et adaptations aux enjeux contemporains (Nord et Sud)

\section{La fabrique des hauts-lieux des alternatives sociales et écologiques dans les marges rurales françaises : le cas de la montagne limousine}

The making of high places of social and ecological aternatives in marginal

French rural areas: the case of plateau de Millevaches

\section{Nassima Hakimi-Pradels}

\section{(2) OpenEdition}

\section{Journals}

\section{Édition électronique}

URL : https://journals.openedition.org/belgeo/48884

DOI : $10.4000 /$ belgeo.48884

ISSN : 2294-9135

Éditeur :

National Committee of Geography of Belgium, Société Royale Belge de Géographie

\section{Référence électronique}

Nassima Hakimi-Pradels, «La fabrique des hauts-lieux des alternatives sociales et écologiques dans les marges rurales françaises : le cas de la montagne limousine », Belgeo [En ligne], 2 | 2021, mis en ligne le 11 juin 2021, consulté le 21 septembre 2021. URL : http://journals.openedition.org/belgeo/ 48884 ; DOI : https://doi.org/10.4000/belgeo.48884

Ce document a été généré automatiquement le 21 septembre 2021.

Belgeo est mis à disposition selon les termes de la licence Creative Commons Attribution 4.0 International. 


\title{
La fabrique des hauts-lieux des alternatives sociales et écologiques dans les marges rurales françaises : le cas de la montagne limousine
}

\author{
The making of high places of social and ecological aternatives in marginal \\ French rural areas: the case of plateau de Millevaches
}

Nassima Hakimi-Pradels

1 Cet article repose sur une analyse empirique d'un espace rural de marge : la montagne limousine située au nord-ouest du Plateau de Millevaches, entre Creuse, Corrèze et Haute-Vienne ${ }^{1}$. Il s'agit d'une marge au sens "d'espace éloigné d'un pôle urbain dynamique de grande taille ", avec une faible densité de population (12 hab./ $/ \mathrm{km}^{2}$ à l'échelle du Parc Naturel Régional de Millevaches), peu accessible par d'autres moyens de locomotion que la voiture et à revenu médian relativement bas (le revenu médian par unité de consommation s'élevait à 17656 euros/an en 2013 pour les communes du Parc Naturel Régional de Millevaches contre 20621 euros/ans à l'échelle nationale à la même date). C'est aussi un espace peu investi par les acteurs économiques majeurs. Pour les pouvoirs publics, il fait partie des territoires qualifiés « d'hyper ruraux ${ }^{2}$ ».

2 Néanmoins, la marge n'est pas la seule représentation à associer à la montagne limousine. Il est souvent postulé qu'il s'agit d'un haut-lieu des alternatives sociales et écologiques par des militants alternatifs (Astier, 2016), des médias (Bordenet, 2018) et des universitaires (Hervieu-Léger, Hervieu, 1979 ; Rouvière, 2016a ; Tommasi, 2018). Il s'agit sans conteste d'un territoire dense en initiatives alternatives, entendues comme des projets dont les fondateurs et la majorité des usagers ont des visions, des pratiques et des modes de relations qui sont disruptifs par rapport aux visions, aux pratiques et aux modes de relations dominants de nos jours et généralement attribués au système de fonctionnement des sociétés capitalistes contemporaines (Koop, à paraître). Cela va des projets en agroécologie aux pompes funèbres alternatives en passant par les garages solidaires, les écoles alternatives et les ressourceries. Sur la montagne 
limousine, nous avons recensé plus de 70 projets de ce type ${ }^{3}$ pour une vingtaine de communes et environ 12000 habitants (avec des chiffres particulièrement élevés pour certains villages comme celui de Faux-la-Montagne où l'on en compte plus de 27 pour 389 habitants). Cette densité des initiatives alternatives sur la montagne limousine interpelle et donne envie de comprendre les processus qui ont permis d'aboutir à une telle situation.

Figure 1. Localisation des initiatives alternatives sur la montagne limousine et année de création.

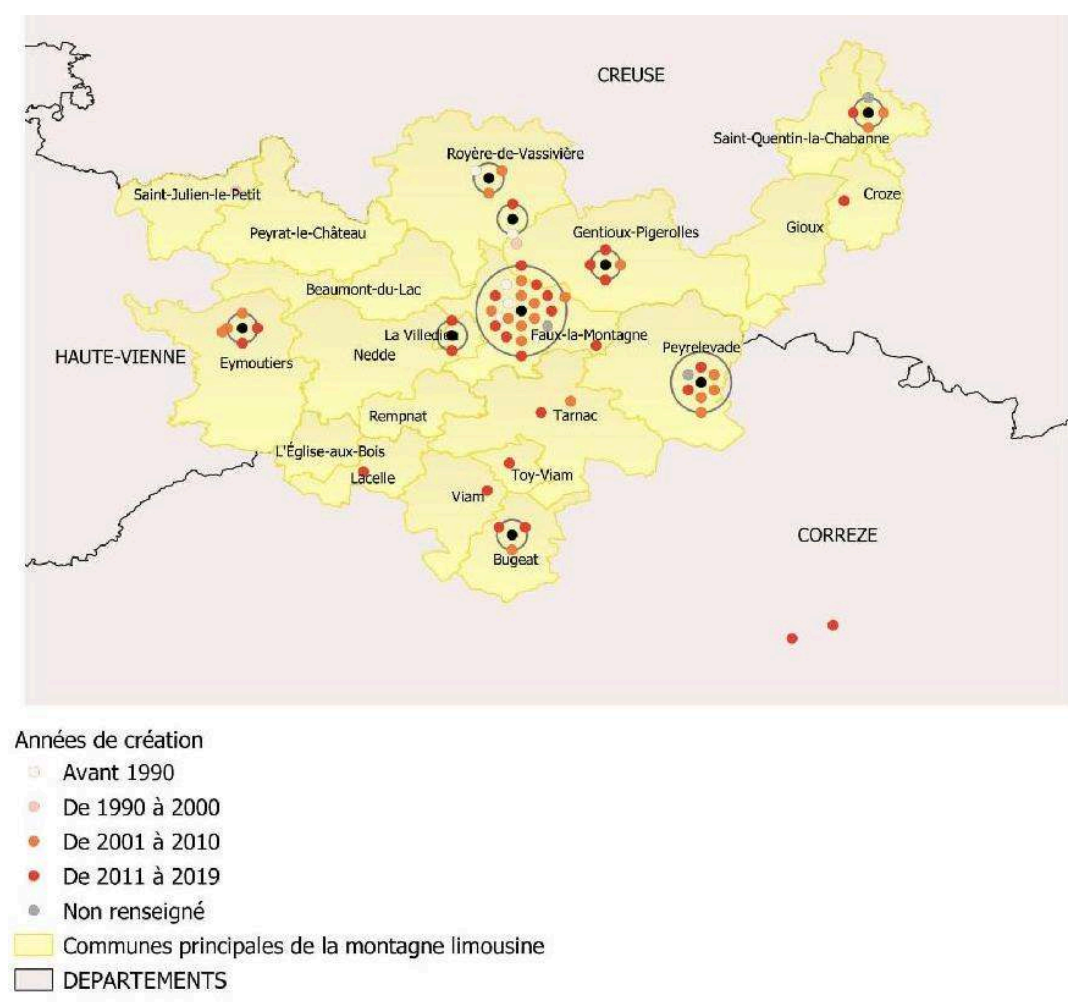

Dans les médias comme dans la littérature scientifique, il est aussi souvent postulé que ces initiatives alternatives seraient amenées de l'extérieur par des néo-ruraux qui s'installent à la campagne pour mettre en acte leur utopie (Rouvière, 2016b ; Tommasi, 2018; Truong, 2020). D’après les sociologues Danièle Hervieu-Léger et Bertrand Hervieu, certains néo-ruraux eux-mêmes partagent cette idée (Hervieu-Léger, Hervieu, 1979). Or sur la montagne limousine tout comme sur d'autres marges montagnardes tels que le plateau du Trièves ou en Ariège, le processus de fabrication de ces hautslieux des alternatives sociales et écologiques est plus complexe. À mon sens, on ne peut pas simplement le comprendre comme un phénomène de développement exogène. $\mathrm{Ce}$ hiatus entre la littérature et l'expérience de terrain nous a amenée à nous poser un certain nombre de questions : quels processus ont conduit à ces situations de très forte densité d'initiatives disruptives par rapport au fonctionnement des sociétés capitalistes actuelles dans certaines marges rurales françaises? Quels acteurs ont joué un rôle dans ces processus? En ayant recours à quels moyens? Et, plus largement, qu'est-ce que ce constat nous apprend sur les transformations sociales en cours dans les espaces ruraux français en marge?

Dans un premier temps, nous soulignerons que la recherche s'est peu intéressée à la compréhension des mécanismes ayant permis d'aboutir à cette situation particulière. 
Ensuite, dans un second temps, nous rendrons compte de la diversité des pratiques en décalage avec les normes dominantes ${ }^{4}$ que l'on a pu constater sur la montagne limousine. Cette diversité oblige à considérer trois facteurs explicatifs de la forte proportion de ces pratiques sur le terrain d'étude : un facteur contextuel, un facteur géographique et un facteur relationnel. D'une part, cette forte proportion s'explique par un contexte global de prise de conscience écologique. D'autre part, elle est liée aux caractéristiques de ce territoire de moyenne montagne de marge. Enfin, elle est facilitée par la capacité de certains acteurs du terrain à tisser des liens entre eux, mais aussi avec des acteurs situés dans des espaces parfois très éloignés.

\section{Les modalités de construction des hauts-lieux des alternatives sociales et écologiques, une question peu étudiée dans la littérature scientifique}

5 Dans la littérature scientifique, on trouve quelques travaux qui essaient de définir ce qu'est le mode de vie alternatif pour un individu ou un groupe (Dubertrand, 2018; Pruvost, 2013; Rouvière, 2016b). Geneviève Pruvost définit par exemple ce qu'elle nomme les alternatifs écologiques "par une alimentation biologique, un habitat partiellement ou totalement écoconstruit, une défense de l'ancrage local et des circuits courts de distribution (en opposition à "l'économie verte" pratiquée par les grands groupes), et des pratiques d'éducation et de médecine alternatives ». Par contre, il y a peu d'études qui tentent de saisir ce qu'est un haut-lieu des alternatives sociales et écologiques et comment il se fabrique. Dans le monde anglophone, on peut citer les travaux de Noël Longhurst qui, dans son article intitulé «The Emergence of an Alternative Milieu: Conceptualising the Nature of Alternative Places» (Longhurst, 2013), explique l'émergence d'un milieu alternatif autour de la ville de Totnes au Royaume-Uni. Malgré cette entrée territoriale, il n'aborde dans son texte qu'un type de population de son terrain : les personnes au mode de vie alternatif assumé, réfléchi et intentionnel. Les autres habitants ne sont pas mentionnés. Concernant la France, les travaux qui s'intéressent aux hauts-lieux des alternatives sociales et écologiques ne sont pas nombreux (Rouvière, 2016b; Tommasi, 2018). Parmi ceux qui ont été produits, on retrouve toutefois une idée commune: dans ces espaces, l'alternative viendrait des néo-ruraux qui s'y sont installés par vagues successives depuis la fin des années 1960 (Rouvière, 2016a; Tommasi, 2018). On serait là face à un phénomène de développement exogène mis en œuvre par des acteurs dotés d'intentions précises et qui cherchent à mettre en acte leur utopie. Sans nier l'apport essentiel que constituent ces travaux, nous chercherons ici à proposer une lecture différente de la construction de ces hautslieux en interrogeant le rôle de tous leurs acteurs, pas seulement les nouveaux arrivants.

6 Pour cette recherche, nous avons fait le choix d'une approche inductive. Nous ne sommes pas arrivée sur le terrain avec des hypothèses à vérifier : nous avons choisi de laisser celles-ci naître des entretiens avec les habitants et des observations avec l'idée de « laisser parler le terrain ». Nous avons fait aussi le pari d'un regard ethnographique porté sur le quotidien et le micro-local : nous avons partagé des moments de vie avec les habitants, nous avons logé chez certains d'entre eux, nous avons participé à la vie des villages, nous avons fait du bénévolat pour des associations et, dans tous ces moments, nous avons concentré notre attention sur les pratiques routinières, en 
particulier celles qui diffèrent des normes dominantes de nos jours et qui sont généralement attribuées aux sociétés capitalistes contemporaines. Notre démarche se situe donc dans le champ de la géographie du quotidien (Gardiner, 2000 ; Hall, 2019 ; Pain, Smith, 2008) ainsi que dans une perspective proche de celle des géographes qui essaient de comprendre comment certains acteurs tentent de construire un monde post-capitaliste en se focalisant sur leurs pratiques quotidiennes (Chatterton, Pickerill, 2010 ; Schmid, Smith, 2020). Au total, nous avons réalisé une cinquantaine d'entretiens avec des acteurs ayant des profils divers (certains correspondent au profil de l'alternatif (Pruvost, 2013) et participant à des initiatives alternatives, d'autres pas du tout ; certains sont nés sur le territoire, d'autres non; certains se présentent comme politisés, d'autres refusent ce qualificatif, etc.). Nos conversations avec ces interviewés ont porté sur leur rapport à l'alternative, sur les initiatives alternatives qu'ils connaissent autour de leur domicile, et sur les liens qu'ils entretiennent les uns avec les autres et surtout sur leurs pratiques de tous les jours.

7 Cette approche inductive nous a amenée petit à petit à nous positionner en faveur d'une définition large de l'activisme. En effet, contestant une approche trop restrictive de celui-ci, des géographes proposent d'élargir sa définition en y incluant les pratiques, modestes et douces, les «quiet politics» qui œuvrent tout autant que les actions plus démonstratives et bruyantes en faveur du bien-être global des sociétés au niveau social et écologique (Pottinger, 2017). Dans cette catégorie, ces géographes choisissent d'inclure des actions du quotidien comme celles en faveur de la conservation de certaines espèces végétales rares, l'accueil chez soi de personnes migrantes en situation illégale ou encore l'autoproduction de vêtements ou de logements. Ces chercheurs invitent donc à changer de regard sur des pratiques considérées la plupart du temps comme anodines. L'interprétation de ces pratiques que ces chercheurs proposent est donc la suivante: ces pratiques ne sont pas anodines, elles portent un message politique; elles ont un véritable pouvoir de subversion par rapport aux modes capitalistes dominants de production et de consommation que les théoriciens de l'activisme politique ont tendance à sous-estimer. Ces chercheurs ont donc une posture bien particulière : ils invitent à considérer comme subversifs par rapport au modèle dominant les acteurs aux pratiques intentionnellement alternatives aussi bien que les acteurs aux pratiques participant à ce qu'eux, en tant que chercheurs, considèrent être une société plus juste et/ou écologique mais qui n'affichent pas cette intentionnalité alternative ou qui ne la revendiquent pas. Cette approche rejoint la volonté de dépasser la dichotomie entre acteurs alternatifs et conventionnels des travaux sur les systèmes alimentaires territoriaux (Lamine, Garçon \& Brunori, 2019) ${ }^{5}$. Elle nous paraît utile pour comprendre comment on aboutit dans certaines marges rurales à des cas de très fortes densités d'initiatives alternatives. Ainsi, pour comprendre les processus qui ont amené à cette situation, nous proposons de considérer comme subversifs toutes les pratiques et les projets qui s'éloignent des normes dominantes de notre société, quelle que soit la position de leurs initiateurs en ce qui concerne le niveau de conscience politique de ce qu'ils font, quelle que soit la nature de leurs intentions (réelles comme affirmées) et quel que soit le discours que ces personnes tiennent à ce sujet (revendicatif ou non notamment). 


\section{La diversité des pratiques qui diffèrent des normes dominantes sur la montagne limousine}

8 La première observation que notre immersion sur le terrain de la montagne limousine a permis de réaliser, c'est qu'on y trouve quatre grands types de pratiques éloignées des normes dominantes. Ces quatre types différents les uns des autres relèvent de trois critères: leur ancienneté sur le territoire, leur niveau de décalage avec les normes dominantes et leur relation avec les manques ou les besoins du territoire.

1. Des activités qui réinvestissent des pratiques qui existent déjà sur le terrain d'étude (ou qui y ont existé) mais en les accompagnant de manières de faire et/ou d'un sens en décalage avec les valeurs dominantes. C'est le cas des ressourceries le Monde Allant Vers à Eymoutiers et Court-Circuit à Felletin. La pratique de la récupération à la base des ressourceries n'est pas nouvelle, encore moins pour les territoires ruraux pauvres tels que le Limousin. Un des interviewés le rappelle: "Le modèle des ressourceries, il n'est pas innovant parce que des ferrailleurs avant, il en passait dans les campagnes. La récup...Moi je viens d'un milieu plutôt pauvre et rural [...]. Avant, c'était la campagne et mon grand-père, il m'expliquait que les trucs d'occasion, t'en achetais. Plein de vêtements...Quand tu n'avais pas les moyens. Il y avait un marché de l'occasion. Le marché du neuf, il s'est généralisé après-guerre...Mais, avant, ce n'était pas ça. Avec les ressourceries, on reprend des trucs qui étaient déjà à l'œuvre auparavant ». Toutefois, si les ressourceries réinvestissent des pratiques existantes, leurs fondateurs leur donnent un nouveau sens, une nouvelle intentionnalité et une nouvelle finalité : leur idée est à la fois d'expérimenter un mode de travail qui diverge des normes de la France d'aujourd'hui puisqu'il s'agit de deux coopératives autogestionnaires et de participer à la diffusion de pratiques écologiques de réparation et d'achats de seconde main, qui ne sont pas non plus la norme dans les sociétés capitalistes contemporaines. Sur ce dernier aspect, on peut considérer que ces personnes s'inscrivent cette fois dans des "prefigurative politics» (Autin, 2016; Chatterton, Pickerill, 2010 ; Yates, 2015). Ce concept désigne la démarche des personnes qui essaient de mettre en actes à leur niveau une vision sociétale alternative. Parmi les principes des politiques préfiguratives figurent la primauté du collectif sur l'individu, la prise de décision horizontale et consensuelle, le refus des hiérarchies, l'antiautoritarisme et, pour certains mouvements, également l'autogestion et l'anticapitalisme. Or on trouve effectivement ces principes dans la plupart des initiatives de notre terrain d'analyse.

2. Des pratiques complètement nouvelles pour le terrain d'étude, qui ont avant tout été mises en place pour répondre à un manque du territoire tout en étant l'occasion d'expérimenter des modes d'organisation en décalage avec les normes dominantes. Le cas du Garage Associatif et Solidaire En Limousin (G.A.S.E.L) qui a ouvert en 2016 sur la commune de Lacelle relève de cette catégorie. À l'origine du projet, il y a eu un faisceau de circonstances et de trajectoires : d'un côté, un membre, aujourd'hui très actif de l'association, souhaitait se lancer dans une aventure collective hors du salariat; de l'autre côté, un groupe d'habitants du bassin de vie de Lacelle voulait apprendre la mécanique car il y a peu de garagistes sur place et ceux qui existent pratiquent des prix jugés trop élevés. L'association que ce groupe a créée a pour but de permettre à chaque adhérent de se former et de prendre en charge l'entretien et les réparations de ses véhicules et engins motorisés dans un esprit de 
solidarité : le coût de l'adhésion est proportionnel aux revenus de l'adhérent. Avec cette initiative, le groupe répond donc à un besoin du territoire tout en expérimentant des manières différentes de faire par rapport aux normes dominantes, notamment concernant le travail, la formation, la consommation et la logique du marché.

3. Des pratiques dont l'origine découle explicitement de la volonté de ne pas se conformer aux normes dominantes sans liens avec les manques du territoire. Le choix de faire partie d'un collectif de vie en est un exemple. Nous avons identifié quatre collectifs aujourd'hui sur la montagne limousine. Un de ces collectifs date de la fin des années 1980, les autres sont vraisemblablement nés après l'an 2000. Les membres de ces collectifs se sont installés sur la montagne limousine avec l'intention explicite de « vivre autrement ». Leur profils sont proches des alternatifs écologiques auxquels s'est intéressée Geneviève Pruvost (2013). Concernant leur organisation, elle varie d'un collectif à l'autre: certains ont acheté un terrain et ont bâti leur habitat dessus, d'autres sont devenus propriétaires d'une ou plusieurs maisons, d'autres encore louent leur logement. Leur point commun réside dans la mise en commun des ressources économiques et le partage des tâches selon un calendrier établi à l'avance. Dans tous les collectifs, on retrouve aussi une part d'autoproduction que cela soit pour la construction de l'habitat, la nourriture ou encore le chauffage.

Une autre pratique qui tranche avec les normes dominantes que l'on peut trouver sur la montagne limousine est l'habitat léger, en yourte généralement. Le choix de l'habitat léger est aussi parfois lié à des motifs économiques et il est difficile de distinguer ce qui relève du désir de "vivre autrement " de la contrainte financière concernant cette pratique. D'autres exemples seraient le choix de ne pas scolariser ses enfants, la pratique du prix libre lors de certains événements ou dans certains lieux de restauration.

13 4. Certaines pratiques qui ont perduré de manière assez importante sur le terrain d'étude alors qu'ailleurs elles sont devenues moins courantes sont aussi en décalage avec les normes dominantes. Les habitants qu'elles concernent s'y livrent le plus souvent sans avoir conscience que d'autres les considèrent comme jouant un rôle pour le bien-être global de la société dans son ensemble. D'autres fois, ils en ont conscience, mais ils choisissent de ne pas les inscrire dans cette intentionnalité, du moins pas en premier lieu. Cela concerne notamment toutes les activités d'autoproduction comme le fait de réaliser ses conserves soi-même, d'avoir un potager, de faire du compost, de construire son logement, d'acheter des aliments directement à leurs producteurs, d'avoir recours à des plantes plutôt qu'à la chimie pour se soigner, etc. Sur la montagne limousine, ces pratiques sont fréquentes sans être toujours associées à un discours du « vivre autrement » ou de « subversion du capitalisme » mais parfois aussi à des considérations d'ordre économique ou à une forme de tradition héritée de ses parents ou de ses grands-parents. C'est peut-être aussi là une forme de «quiet politics" (Pottinger, 2016) : elles jouent un rôle pour le bien-être global de la société dans son ensemble sans pour autant avoir été réalisées dans ce but. Les habitants du terrain d'étude dont le profil se rapproche de l'alternatif de Genevieve Pruvost (2013), n'utilisent pas cette dénomination de " pratique préfigurative » qui est aujourd'hui plutôt restreinte à la recherche mais, systématiquement, le regard qu'ils jettent sur ces pratiques est extrêmement positif sans doute car elles s'alignent bien avec leurs propres intentions et leurs propres manières d'agir. 
14 Ainsi, sur la montagne limousine, des pratiques en décalage avec les normes dominantes ont perduré, se sont développées et se développent encore. Elles sont à la fois nombreuses et très diverses. Contrairement à ce que l'on pourrait penser intuitivement, elles ne sont pas uniquement le fait de néo-ruraux à l'ethos alternatif (Pruvost, 2013). Elles sont aussi le fait de personnes qui ne relèvent pas de cet ethos et qui agissent sans s'inscrire dans cette intentionnalité.

Ce constat nous a permis de construire une hypothèse : si la montagne limousine est devenue un haut-lieu des initiatives et des pratiques en rupture avec les normes dominantes, ce n'est pas uniquement le fait de l'installation de néo-ruraux à l'ethos alternatif. C'est davantage un mélange entre des pratiques menées par des personnes ayant cet ethos et des pratiques menées par des personnes qui ne correspondent pas à ce profil.

On ne peut alors que s'interroger sur les raisons de cette proportion importante de pratiques et d'initiatives différant des normes dominantes sur la montagne limousine. Sur ce point, l'immersion sur le terrain nous permet de proposer une autre hypothèse encore, celle que l'importance de ces initiatives et de ces pratiques sur la montagne limousine peut s'expliquer par trois facteurs: un facteur contextuel, un facteur géographique et un facteur relationnel.

\section{Trois facteurs explicatifs de la proportion importante de « pratiques différentes » sur la montagne limousine}

\section{Le contexte : un moment de prise de conscience collective des limites du mode de vie moderne}

17 Le premier facteur explicatif de la proportion importante " des pratiques différentes" sur la montagne limousine est d'ordre contextuel : pour comprendre ce phénomène, il faudrait le relier au contexte mondial actuel où la conscience collective des limites du mode de vie moderne a pris de l'ampleur et a changé de dimension. En effet, ces dernières années, les évènements de nature à faire penser que notre système capitaliste mondial n'est pas soutenable du point de vue social et écologique se sont multipliés et de nombreuses voix scientifiques se sont élevées pour en parler (Boltanski, Chiapello, 2011; Escobar, Andrade Pérez \& Bednik, 2018 ; Harvey, 2014 ; Latour, 2017 ; Piketty, 2019). Les problèmes que nous rencontrons sont nombreux : le changement climatique, le dépassement des limites de soutenabilité de la biosphère, la raréfaction des ressources naturelles, l'augmentation des inégalités sociales, des mouvements migratoires inédits, des crises de gouvernance, etc. En conséquence, et même si elles sont loin d'être majoritaires, les personnes ayant des convictions et des discours critiques en faveur d'un changement de mode de vie sont de plus en plus nombreuses. Certains les accompagnent d'actes, d'autres non. On peut se poser la question de savoir s'il s'agit d'un nouveau mouvement social en cours de construction (Melucci, 2016; Touraine, 1980).

18 Cette prise de conscience collective peut expliquer en partie l'amplification «des pratiques différentes » sur la montagne limousine ces dernières années. En effet, s'il est difficile de l'évaluer avec précision, il est clair que, parmi les personnes arrivées depuis peu sur le plateau de Millevaches, on compte une proportion importante d'individus 
que l'on peut rattacher à ce mouvement récent. En s'installant, ces personnes viennent grossir le nombre de "pratiques différentes" sur la montagne limousine, plus précisément elles viennent grossir le nombre de «pratiques intentionnellement différentes ». Par exemple, un couple d'une trentaine d'années avec lequel j'ai pu avoir un entretien correspond à cette catégorie. L'homme est un enfant de néo-ruraux de la montagne limousine qui est parti vivre en ville au moment de ses études supérieures. La femme a grandi en région parisienne. Ils se sont rencontrés dans une grande métropole française et y ont vécu quelques années. À partir de 2015 s'est élaboré petit à petit leur projet de vivre sur le plateau, dans un village proche de celui des parents $d u$ jeune homme. Chez eux, le désir de vivre sur la montagne limousine est assez clairement associé à l'envie d'avoir un nombre plus important de pratiques en cohérence avec leurs valeurs. Celles-ci portent sur l'envie d'avoir un rapport à la nature et au corps différents des normes dominantes mais aussi un autre rapport au genre, à l'éducation, à la consommation, au travail, à la hiérarchie, à la science, à la médecine, etc. Dans la ville où ils ont vécu ensemble, ils avaient quelques " pratiques différentes » mais, d'après eux, habiter sur la montagne limousine leur permet d'en avoir bien davantage. Pour m'expliquer cette idée, la jeune femme m'a dit plus précisément ceci : "Ici je suis beaucoup plus en raccord avec ce que je veux être, ma façon de vivre et tout. Dans la ville où l'on habitait avant, c'est arrivé qu'on choppe des amap ${ }^{6}$ ou des machins comme ça, mais ici tu te sens plus portée par des valeurs que tu partages. Du coup, c'est beaucoup moins compliqué. Tu es moins obligée de te justifier ou t'es moins obligée de ne pas dire que tu fais quelque chose de peur que la personne en face ne te comprenne pas ou que tu prennes une heure pour lui expliquer ce que tu fais et pourquoi tu le fais. C'est plus facile». Depuis leur installation sur la montagne limousine, ces deux personnes ont pu y auto-construire une maison écologique ; elles se sont aussi intégrées à des groupes de travail sur le sujet de la communication nonviolente, notamment en ce qui concerne les relations parents-enfants. La femme a décidé d'accoucher à la maison et pas à l'hôpital à la suite de discussions avec d'autres habitantes de la montagne, etc. Selon ce couple, le mode de vie de la ville ne permettait pas de bien vivre, d'une part car il ne leur offrait que peu de possibilités de mettre en acte leurs valeurs telles que l'écologie et l'autonomie et, d'autre part, parce qu'il les amenait à avoir des comportements qu'ils jugeaient mauvais pour eux comme de ne pas garder assez de temps pour des activités autres que le travail salarié.

L'installation très récente de ce genre de couple sur la montagne limousine ces toutes dernières années peut être reliée au contexte global qui fait que, chez les jeunes générations en particulier, on constate une proportion forte et croissante de personnes conscientisées sur la non-soutenabilité de nos modes de vie. Parmi ces personnes, certaines font le choix d'aller vivre dans des espaces ruraux. Au sein de ces espaces ruraux, ceux qui, comme la montagne limousine, sont réputés pour leur image dynamique, contestataire et alternative, sont particulièrement prisés.

Toutefois, comme ailleurs, on ne trouve pas chez tous les habitants de la montagne limousine exactement les mêmes discours critiques : il existe des différences au niveau des idées. Parfois ces divergences portent sur la remise en question de nos modes de vie actuels mais pas uniquement. Parfois, ces différences rejouent l'opposition entre néoruraux alternatifs et « anciens habitants » (Tommasi, 2018) mais d'autres fois, elles s'en détachent complètement. Par contre, au niveau des pratiques de la vie quotidienne, il y a beaucoup moins de divergences. Dans de nombreux domaines, les pratiques des acteurs de la montagne limousine se rejoignent. Comment expliquer ce phénomène? 
Une hypothèse serait de le relier à un élément d'ordre géographique : le fait de vivre dans un espace rural de marge.

\section{La géographie : quelles relations entre les " pratiques différentes » et la marge ?}

Sur la montagne limousine, on trouve « des pratiques différentes » réalisées par deux types d'acteurs: d'une part des acteurs qui sont très conscients des enjeux de soutenabilité et qui réalisent ces pratiques de manière intentionnelle et, d'autre part, des acteurs qui n'ont pas ce niveau de conscience et/ou qui s'inscrivent dans d'autres intentionnalités. Toutefois, et malgré leurs différences, ces deux types d'acteurs sont, par leurs "pratiques différentes », engagés dans le "vivre autrement». On peut dès lors émettre l'hypothèse d'un lien entre le fait d'avoir des "pratiques différentes » et celui d'habiter une marge. En effet, ne serait-ce pas une caractéristique de la marge que d'être favorable à l'existence de pratiques disruptives par rapport aux normes dominantes ??

Dans le cas de ceux qui ne donnent pas de sens politique ou subversif à leurs actions, leurs pratiques peuvent s'expliquer par le fait que leur territoire de vie ait été moins que d'autres façonné par la modernité (et un de ses principaux corollaires, le capitalisme); certaines pratiques devenues habituelles pour quelqu'un qui habite, par exemple, une grande métropole n'y sont pas devenues la norme. En conséquence, des pratiques anciennes ont pu perdurer, et de la place a été laissée pour des "pratiques différentes » qui ont vu le jour plus récemment. Ainsi ces personnes ont des pratiques que certains habitants engagés intentionnellement dans le "vivre autrement" effectuent aussi mais en leur donnant un autre sens. Par exemple, si les deux types d'acteurs ont tendance à acheter en vente directe leurs produits alimentaires, certains associent cette pratique à des préoccupations écologiques tandis que d'autres n'ont pas cette raison en tête, ou, du moins, pas en premier lieu. Il en est de même dans plusieurs autres domaines, par exemple pour l'achat de seconde main ou le fait de fabriquer soimême certains produits comme le pain ou ses vêtements. Cet ensemble de phénomènes permet en fin de compte aux deux catégories d'acteurs de se rejoindre sur certaines pratiques et donc parfois de se rencontrer, d'échanger, de s'entraider et même de construire des projets communs.

Par ailleurs, si les acteurs conscientisés et porteurs de discours critiques de la montagne limousine et les habitants de cet espace rural qui ne le sont pas (ou moins) se rejoignent sur certaines pratiques, c'est aussi parce que leur cadre de vie, leur environnement, est le même et qu'il influe sur leurs pratiques. Ainsi, habitant le même territoire montagnard de marge, ils sont confrontés aux mêmes contraintes (qu'elles soient liées à la météo, aux possibilités de déplacement ou à la présence des services d'intérêt général ou de la culture par exemple) et les pratiques qui permettent de les surmonter sont consensuelles, qu'elles soient ou non la norme ailleurs que sur la montagne limousine. C'est ainsi par exemple qu'on peut expliquer que, dans certaines initiatives de commandes de produits groupés (soit pour l'alimentation, soit pour le jardinage), on trouve des acteurs qui appartiennent à tous les profils de la montagne limousine. Cette situation peut s'expliquer par des raisons pratiques: pour acheter ces produits, si l'on ne fait pas partie d'un collectif de commandes groupées, il faut avoir une voiture et conduire sur de longues distances. Les commandes groupées permettent 
d'éviter ces déplacements tout en faisant des économies d'argent. Un autre exemple concerne les transports : le garage de Lacelle a du succès auprès des habitants de tout type de la montagne limousine car il y a peu de garagistes sur le territoire et qu'en plus il est intéressant économiquement de s'y rendre. Néanmoins, ce ne sont pas seulement les contraintes de leur cadre de vie que les habitants de la montagne partagent, ce sont aussi les aménités. La présence de la nature et en particulier de la forêt en fait partie. L'attachement à la forêt conduit bon nombre d'habitants du plateau à avoir une attention particulière à la manière dont cette ressource est gérée. Sa gestion actuelle pose en effet plusieurs questions. Celles-ci concernent à la fois les effets néfastes de la monoculture pratiquée sur le plateau, des problèmes de perte de biodiversité, des problèmes de fermeture ou de dégradation des paysages, des enjeux de pollution de l'eau, etc. Lorsque des personnes se mobilisent pour organiser des temps de réflexion sur la forêt, pour critiquer des projets la concernant ou pour proposer d'autres manières de la gérer (avec la sylviculture douce par exemple), elles arrivent à attirer des habitants de la montagne limousine de tous profils. Nous n'entendons pas par-là que les habitants du terrain d'étude sont absolument tous sensibles à l'idée de mettre en place des alternatives forestières. Des tensions existent, notamment avec les habitants qui soutiennent des modes plus classiques de gestion de la forêt (Tommasi, 2018). Néanmoins, il est vrai aussi que dans les moments de rencontres autour de la forêt que j'ai pu observer ${ }^{8}$, on trouve à la fois des alternatifs écologiques (Pruvost, 2013) et des personnes qui n'ont pas les caractéristiques de ce groupe social. Ainsi, à l'occasion de ces évènements, des personnes aux profils sociaux très différents se retrouvent, échangent, organisent parfois des actions de protestation ensemble, montent des projets, etc. Ainsi, sur ce sujet de la forêt, les différents habitants de la montagne se rejoignent aussi sur un certain nombre de moments et de pratiques. Le fait de partager le même cadre de vie (ici, très concrètement, le fait d'habiter sur un plateau très boisé) est un élément qui a son importance pour comprendre ce phénomène.

24 Au final, ces exemples montrent que, sur la montagne limousine, il y a un lien entre la proportion importante de pratiques en décalage avec les normes dominantes dans les sociétés capitalistes contemporaines et les caractéristiques de la marge. En outre, ils révèlent que des liens se tissent entre tous les acteurs de ce territoire au niveau de leurs pratiques et ce, en dépit des différents sens et des différentes intentions auxquels ils les associent. Or, il s'agit là aussi d'un élément qui contribue à la diffusion "des pratiques différentes » sur la montagne limousine.

\section{Le relationnel : la circulation des pratiques passe aussi par le tissage de liens sociaux}

Le troisième facteur explicatif de la croissance des pratiques en décalage avec les normes dominantes sur la montagne limousine est un facteur relationnel. En effet, ces pratiques loin d'être cantonnées à tel ou tel groupe social, circulent à travers le tissage de liens entre les différents habitants de ce territoire, contribuant à en faire un lieu du « vivre autrement ».

Par exemple un agriculteur de la montagne limousine est resté très marqué par sa rencontre avec une jeune femme qui avait décidé d'abandonner son emploi en collectivité territoriale pour se reconvertir dans l'agriculture. Il a beaucoup discuté 
avec elle, notamment de circuits courts et de vente directe. Ils ont essayé de monter ensemble un GAEC (Groupement Agricole d'Exploitation en Commun). Le projet a échoué. Aujourd'hui, ces deux personnes n'ont plus de contact, mais l'agriculteur a tout de même décidé de se lancer dans la vente directe ${ }^{9}$ et la femme a monté un élevage de brebis. Pour l'agriculteur, il ne fait pas de doute que ce sont ces échanges avec cette femme qui l'ont amené à la vente directe car c'est elle qui l'a amené à se former sur cette pratique et qui lui a transmis les outils administratifs et techniques pour le faire. Bien que distendus aujourd'hui, les liens qui ont existé entre ces deux acteurs ont en tout cas à la fois clairement contribué à la diffusion de cette pratique des circuits courts qui n'est pas dominante aujourd'hui sur la montagne limousine et ils ont en même temps permis à une personne de se former à une pratique nouvelle pour elle, l'élevage de brebis.

Un deuxième exemple est un projet d'éco-hameau construit en 2017. A l'initiative de la municipalité, des ateliers ont été organisés autour de ce projet. Des habitants aux profils divers y ont participé : des néo-ruraux récents et anciens, des personnes qui ont toujours habité la montagne, des alternatifs, des non-alternatifs, etc. Au fur et à mesure des rencontres, une décision en décalage avec les pratiques dominantes a été prise : ce projet d'éco-hameau comprendrait de l'habitat léger, probablement des yourtes. Pour un interviewé qui a participé à cet atelier ${ }^{10}$, on a là un exemple réussi de "rencontre entre les mondes " alors même que, concomitamment, on assistait à des conflits dans d'autres communes du plateau autour de cette question de l'habitat léger. En effet, d'après le même interviewé, cette pratique a été adoptée par les villageois pour deux raisons: d'une part, car ils connaissent déjà des personnes qui habitent dans des yourtes situées près de leur commune ; et d'autre part, parce qu'elle répond à un besoin du village d'attirer de nouvelles personnes : «Les habitants de cette commune ont fait le constat qu'accéder ici à l'habitat, c'est compliqué. Que faire construire, c'est cher. Et que tout le monde, en particulier ceux qui viennent s'installer ici, n'ont pas le fric. Et donc, à un moment donné, si on ne fait pas en sorte qu'ils puissent trouver chaussure immobilière à leurs pieds, ben on ne réussira pas à les installer. Et donc, on ratera un des enjeux ici qui est de regonfler démographiquement le coin. Voilà, ils ont fait ce parcours-là. Et donc à un moment donné, ça a percolé. Il y a eu du partage. Il y a une vision commune qui s'est élaborée petit à petit ». Ainsi, grâce aux échanges qui ont eu lieu au cours de ces ateliers mais aussi à la fréquentation quotidienne de personnes aux modes de vie différents, une pratique en décalage avec les normes a été intégrée à un projet porté par la municipalité de manière douce.

Dans ce dernier exemple comme dans le précédent, il transparaît que si « des pratiques différentes " se répandent sur la montagne limousine, leur circulation passe par le tissage de liens entre les habitants.

\section{Conclusion}

Ainsi, notre enquête nous permet d'avancer que si la montagne limousine est devenue un haut-lieu des initiatives et des pratiques en rupture avec les normes des sociétés capitalistes contemporaines, ce n'est pas uniquement le fait de l'installation de néoruraux à l'ethos alternatif. C'est davantage un mélange entre des pratiques menées par des personnes ayant cet ethos et des pratiques menées par des personnes qui ne correspondent pas à ce profil. Quant à l'importance de ces initiatives et de ces pratiques 
sur la montagne limousine, on a posé l'hypothèse qu'elle peut s'expliquer par trois facteurs : un facteur contextuel, un facteur géographique et un facteur relationnel.

De surcroît, et au-delà de ces constats et hypothèses, notre expérience sur le terrain rapportée dans cet article nous invite à penser que des mutations sont en train de s'opérer dans les représentations des espaces ruraux de marge situés en montagne. En effet, une sorte de renversement est en train de s'opérer, ou du moins de glissement, au niveau de la manière d'évaluer l'intérêt des espaces de marges de montagne : d'espaces répulsifs ${ }^{11}$, ils tendent à devenir des espaces chargés plus positivement. La construction d'espaces identifiés comme des hauts-lieux de pratiques alternatives dans ces marges et l'installation choisie d'habitants de plus en plus nombreux et diversifiés (certains à l'ethos très alternatifs (Pruvost, 2013) et d'autres moins ou pas du tout) dans ces campagnes en témoignent bien : alors que pendant longtemps le discours dominant sur ces espaces consistait à dire qu'il s'agissait d'espaces inintéressants et à fuir, notamment pour des jeunes, aujourd'hui les mentalités évoluent et de plus en plus de personnes développent un discours plus positif sur ces espaces, discours dans lequel leur statut de marge devient un atout : être éloignés des centres permet d'être moins touchés par les crises et les problèmes de la société capitaliste dont les grandes villes sont la tête de pont. Qui plus est, être éloignés des centres permet des expérimentations plus difficiles à mettre en place dans d'autres espaces. S'éloigner des normes y est moins difficile. Or, sous l'effet des multiples crises sociales et écologiques qui touchent notre monde, un nombre croissant de personnes développent ces désirs d'éloignement.

Si on élargit la réflexion aux montagnes en général, on constate que cette inversion n’a rien d'inédit. Ce n'est pas la première fois qu'elle se produit. L'historien Luigi Lorenzetti l'a souligné (Lorenzetti, 2019) : les représentations des montagnes ont évolué dans le temps. Si aujourd'hui, elles sont majoritairement perçues comme des périphéries et des espaces plutôt conservateurs, parfois dans le passé, elles ont été considérées comme des centres et des têtes de pont de l'innovation (certaines d'entre elles du moins). Pour décrire le bourg de Chiavenna dans les Alpes lombardes, l'écrivain allemand David Granz utilisait d'ailleurs une expression amusante : "Der Pündtner London », soit " la Londres des Grisons ».

\section{BIBLIOGRAPHIE}

ASTIER M. (2016), Faire revivre les campagnes : en Creuse comme ailleurs, c'est possible, Reporterre.

AUTIN G. (2016), Ouverture des possibles : utopies concrètes et pratiques préfiguratives, Posssibles.

BOLTANSKI L., CHIAPELLO È. (2011), Le nouvel esprit du capitalisme, Paris, Gallimard (Tel), 971 p.

BORDENET C. (2018), « À Faux-la-Montagne, “on ne peut pas se payer le luxe de choisir. Alors, on accueille tous les projets ” ", Le Monde, 17 mai 2018. 
CHATTERTON P., PICKERILL J. (2010), “Everyday activism and transitions towards post-capitalist worlds: Everyday activism and transitions towards post-capitalist worlds”, Transactions of the Institute of British Geographers, 35, 4, pp. 475-490.

DUBERTRAND B. (2018), « Au fond de la montagne...l'utopie : expériences alternatives contemporaines dans la moyenne montagne ariègeoise ", in Montagnes en mouvements. Dynamiques territoriales et innovation sociale (ouvrage dirigé par Marie-Christine Fourny), PUG et UGA Editions.

ESCOBAR A., ANDRADE PEREZ R. \& BEDNIK A. (2018), Sentir-penser avec la Terre : l'écologie au-delà de l'Occident, Paris, Seuil.

GARDINER M.E. (2000), Critiques of everyday life, London, New York, Routledge, 242 p.

HALL S.M. (2019), “Everyday austerity: Towards relational geographies of family, friendship and intimacy", Progress in Human Geography, 43, 5, pp. 769-789.

HARVEY D. (2014), Brève histoire du néolibéralisme, Paris, Prairies ordinaires Editions.

HERVIEU-LEGER D., HERVIEU B. (1979), Le retour à la nature : « Au fond de la forêt ... l'État », Paris, Seuil.

KOOP K. (à paraître), “Escaping from capitalism : Using improvisation to enact alternative lifeworlds in France's mountain regions”, in HALL S., PIMLOTT-WILSON H. \& HORTON J. (dir.), Austerity Across Europe : Living, Feeling and Experiencing Economic Crises, Routledge.

LAMINE C., GARÇON L. \& BRUNORI G. (2019), “Territorial agrifood systems: A Franco-Italian contribution to the debates over alternative food networks in rural areas", Journal of Rural Studies, 68, pp. 159-170.

LATOUR B. (2017), Où atterrir ? comment s'orienter en politique, Paris, La Découverte, 155 p.

LONGHURST N. (2013), "The Emergence of an Alternative Milieu: Conceptualising the Nature of Alternative Places”, Environment and Planning A: Economy and Space, 45, 9, pp. 2100-2119.

LORENZETTI L. (2019), La montagne, objet social et politique. La position périphérique vue sur le temps long, Communication dans le cadre du LabEx ITEM.

MELUCCI A. (2016), « Mouvements sociaux, mouvements post-politiques », International Review of Community Development, 10, pp. 13-30.

NEUMEIER S. (2012), "Why do Social Innovations in Rural Development Matter and Should They be Considered More Seriously in Rural Development Research? - Proposal for a Stronger Focus on Social Innovations in Rural Development Research: Social innovations in rural development", Sociologia Ruralis, 52, 1, pp. 48-69.

NOACK A., FEDERWISCH T. (2019), “Social Innovation in Rural Regions: Urban Impulses and Cross-Border Constellations of Actors: Social innovation in rural regions”, Sociologia Ruralis, 59, 1 , pp. 92-112.

PAIN R., SMITH S. (dir.) (2008), Fear: critical geopolitics and everyday life, Aldershot, Hants, England, Burlington, VT, Ashgate (Re-materialising cultural geography), $256 \mathrm{p}$.

PIKETTY T. (2019), Capital et idéologie, Paris, Éditions du Seuil (Les livres du nouveau monde), $1197 \mathrm{p}$.

POTTINGER L. (2017), "Planting the seeds of a quiet activism", Area, 49, 2, pp. 215-222.

PRUVOST G. (2013), « L'alternative écologique : Vivre et travailler autrement », Terrain, 60, pp. 36-55. 
ROUVIERE C. (2016a), Retourner à la terre : l'utopie néo-rurale en Ardèche depuis les années 1960, Rennes, Presses Universitaires de Rennes.

ROUVIERE C. (2016b), « Migrations utopiques et révolutions silencieuses néorurales depuis les années 1960 », Cahiers d'histoire. Revue d'histoire critique, 133, pp. 127-146.

SCHMID B., SMITH T.S. (2020), "Social transformation and postcapitalist possibility: Emerging dialogues between practice theory and diverse economies", Progress in Human Geography, doi: $10.1177 / 0309132520905642$.

TOMMASI G. (2018), « Innovations sociales et mobilisations dans la montagne limousine », in Les espaces ruraux en France, Atlande.

TOURAINE A. (1980), La prophétie anti-nucléaire, Paris, Seuil (Sociologie permanente), 373 p.

TRUONG N. (2020), « La France des oasis » (série en 6 volets), Le Monde, août 2020.

YATES L. (2015), “Rethinking Prefiguration: Alternatives, Micropolitics and Goals in Social Movements", Social Movement Studies, 14, 1, pp. 1-21.

\section{NOTES}

1. Nous présenterons ici une étude de cas fondée sur une enquête de terrain effectuée entre 2017 et 2018 dans le cadre du LabEx ITEM (Université Grenoble Alpes).

2. Cf. le rapport de 2014 du sénateur Alain Bertrand intitulé « Hyper-ruralité : un pacte national en 6 mesures et 4 recommandations pour restaurer l'égalité républicaine ».

3. Ce recensement se base sur des recherches sur internet et la consultation régulière de journaux locaux depuis janvier 2017. De surcroit, pour construire ce recensement, nous avons effectué deux périodes longues d'observation en immersion sur le terrain au cours desquelles une cinquantaine d'entretiens semi-directifs avec des habitants divers du terrain d'étude ont été effectués (en totalité, nous avons habité plus de 3 mois sur le terrain, à quoi l'on peut ajouter quelques visites plus brèves pour réaliser des entretiens ou participer à un événement précis).

4. Pour des questions d'aisance d'écriture et de lecture, nous utiliserons parfois dans ce texte l'expression de «normes dominantes » pour désigner « les normes qui dominent de nos jours et qui sont généralement attribuées aux sociétés capitalistes contemporaines ».

5. Les travaux sur les systèmes alimentaires territoriaux se cantonnent néanmoins uniquement aux questions alimentaires et agricoles alors que l'approche développée dans cet article est intersectorielle.

6. Association pour le Maintien d'une Agriculture Paysanne.

7. Nous n'ignorons pas qu'il existe des espaces alternatifs au sein d'autres espaces que ceux dont il est question dans l'article, notamment au sein des espaces métropolitains. Nous ne postulons pas que les marges rurales sont les seuls espaces favorables à l'existence de pratiques en rupture par rapport aux normes qui dominent de nos jours et qui sont généralement attribuées aux sociétés capitalistes actuellement en crise. Nous émettons seulement l'hypothèse que certaines caractéristiques des marges les rendent favorables à ce type de pratiques. Mais elles n'ont pas l'exclusivité et d'autres espaces, notamment à l'échelle infra-urbaine, constituent aussi des terreaux favorables pour ces types de pratiques, pour d'autres raisons.

8. En 2018, le sujet de la forêt était assez présent sur la montagne limousine. Un réalisateur habitant sur la montagne limousine, François-Xavier Drouet, a réalisé un film intitulé Le temps des forêts et plusieurs projections et discussions ont été organisées autour de son travail. Par ailleurs, un projet d'usine de pellets torréfiés (granulés issus du bois-énergie) dans une des communes du plateau a suscité un mouvement de mobilisation contre lui. Cela a aussi eu pour conséquence que 
l'on parle beaucoup de la forêt à ce moment-là. Diverses réunions et moments d'échange ont été organisés autour du projet et plus généralement de la forêt. En outre, à l'occasion de la Fête de la Montagne limousine de 2018, un forestier de la montagne limousine a eu l'opportunité de faire une conférence et un temps de discussion sur la forêt sur le plateau de Millevaches, etc.

9. Avant, cet agriculteur n'élevait que des broutards: après leur sevrage, ces jeunes veaux partaient en Italie pour les étapes de l'engraissement et de l'abattage. Depuis quelques années, il engraisse lui-même ces animaux et s'occupe de les vendre en vente directe.

10. Entretien numéro 5, octobre 2017.

11. Aujourd'hui, dans l'imaginaire de la plupart des gens en Occident, dans l'imaginaire dominant, les territoires ruraux de marge situés en moyenne montagne sont identifiés par des caractéristiques négatives : ils sont loin des centres et de leurs aménités, ils sont peu accessibles par les transports collectifs, il est difficile d'y circuler sans voiture, on y observe une faible activité économique, les services publics et plus généralement les services d'intérêt général y sont de moins en moins importants, les écoles ferment, il y a peu de médecins, les bureaux de poste se comptent sur les doigts d'une main, etc.

\section{RÉSUMÉS}

Concernant la construction des hauts-lieux des alternatives sociales et écologiques en milieu rural tels que le Larzac, le Couserans ou bien le plateau de Millevaches, il est souvent postulé que les alternatives y seraient amenées de l'extérieur par des néo-ruraux qui s'installent à la campagne pour mettre en acte leur utopie. L'objectif de cet article est de nuancer cette thèse en développant l'idée selon laquelle, dans ces hauts lieux des alternatives, les initiatives et les pratiques en décalage avec les normes dominantes ne sont pas uniquement le fait de néo-ruraux à l'ethos alternatif, très conscients des enjeux de soutenabilités et œuvrant intentionnellement à construire une société qu'ils veulent plus juste et plus écologique mais aussi de personnes qui n'ont pas ce niveau de conscience et/ou qui s'inscrivent dans d'autres intentionnalités. Cette nuance ayant été apportée, il sera ensuite proposé d'explorer trois facteurs explicatifs de la forte proportion de ce type spécifique d'initiatives et de pratiques dans certains espaces ruraux français de marge : un facteur contextuel, un facteur géographique et un facteur relationnel.

Larzac, Couserans or plateau de Millevaches are remote rural highlands. During the $\mathrm{XX}^{\text {th }}$ century, they became famous for being high places of social an ecological aternatives. This transformation is usually seen as a process brought in by outsiders: neo-rural people who settle in the countryside to act out their utopia. The goal of this article is to discuss this thesis by developing the idea according to which, in these high places of alternatives, initiatives and practices breaking with the norms of contemporary capitalist societies are not solely made by neo-rural people with an alternative ethos, very aware of the challenges of sustainability and working intentionally to build a society that they want more just and more ecological but also by people who do not have this awareness and/or who act with other motives. In the second part, three explanatory factors will be explored to explain the high proportion of this specific type of initiatives and practices in certain marginal French rural areas: a contextual factor, a geographical factor and a relational factor. 
INDEX

Mots-clés : alternatives sociales et écologiques, hauts-lieux, durabilité, écologie, espaces ruraux, montagnes, marges, néo-ruraux, Limousin

Keywords : social and ecological alternatives, high places, sustainability, ecology, rural areas, mountains, margins, neo-rural people, Limousin

\section{AUTEUR}

\section{NASSIMA HAKIMI-PRADELS}

Agrégée de géographie et doctorante, LabEx ITTEM (Innovations et Transitions Territoriales en Montagne), Laboratoire Pacte, Université Grenoble Alpes (UGA), nassima.hakimi@laposte.net 